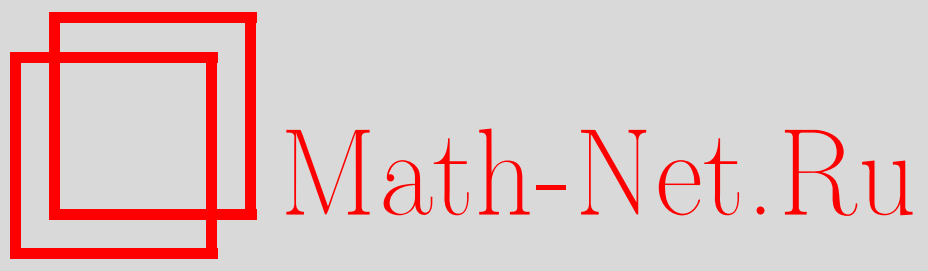

А. М. Вершик, Задача о центральных мерах на пространствах путей градуированных графов, Функи. анализ и его прил., 2014, том 48, выпуск 4, 26-46

DOI: https://doi.org/10.4213/faa3166

Использование Общероссийского математического портала MathNet.Ru подразумевает, что вы прочитали и согласны с пользовательским соглашением http://www . mathnet.ru/rus/agreement

Параметры загрузки:

IP : 54.205 .225 .156

26 апреля 2023 г., 02:28:33

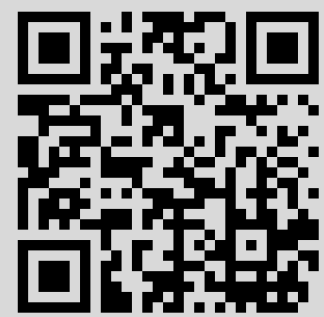




\title{
Задача о центральных мерах на пространствах путей градуированных графов*
}

\author{
(c) 2014. А. М. ВЕРшик
}

В работе предлагается новый метод описания инвариантных мер на марковских компактах и на пространствах путей графов и, тем самым, описания характеров некоторых групп и следов $A F$-алгебр. Метод использует свойства фильтраций, ассоциированных с графом и, в частности, понятие стандартной фильтрации. Основным средством служит вводимая на симплексах мер внутренняя метрика, являющаяся итерированной метрикой Канторовича, а центральный результат состоит в том, что относительная компактность в этой метрике гарантирует конструктивное перечисление инвариантных эргодических мер. В числе приложений - ряд классических теорем об инвариантных мерах.

\section{§1. Введение}

Целью этой работы является точная формулировка нового способа нахождения эргодических мер на марковских компактах или на пространстве путей диаграммы Браттели, инвариантных относительно хвостового разбиения. Способ предполагает наличие некоторого условия равномерной компактности относительно вводимых метрик, и если оно выполнено, то полный список инвариантных эргодических мер параметризуется точками компактификации. Эргодичность меры в данном случае означает, что мера удовлетворяет закону 0-1 относительно хвостовой фильтрации. Предлагаемый метод представляет собой развитие и усиление эргодического метода нахождения инвариантных эргодических мер, который основан на эргодической теореме или на теореме о сходимости мартингалов и не требует других условий. Вследствие этого он может быть применен для любой аппроксимации инвариантных мер. Но именно из-за этого его использование привносит серьезные трудности в вычислениях конечномерных распределений.

Главная трудность в эргодическом методе состоит даже не столько в вычислениях, сколько в доказательствах полноты списка. Но оказывается, что во многих естественных ситуациях, таких, как различные обобщения теоремы де Финетти, теоремы о характерах бесконечной симметрической группы и др., хвостовое разбиение устроено так, что его естественная аппроксимация обладает гораздо более сильными свойствами, чем просто сходимость почти всюду или по мере. А именно, хвостовая фильтрация оказывается стандартной в смысле теории фильтраций, что, в частности, означает сходимость аппроксимации в очень сильном смысле. По-другому это формулируется так: во многих примерах инвариантные меры обладают скрытыми обобщениями свойства независимости, а стандартность по существу и есть обобщенная независимость. Фактически настоящая работа есть результат применения теории фильтраций, которой автор

*Работа поддержана грантом РНФ 14-11-00581. 
занимался, начиная с 60-х гг., к вопросам, близким к теории представлений локально конечных групп, их характеров и комбинаторике в целом. Следующее ниже изложение, тем не менее, замкнуто в себе и не требует обращения к другим работам. Мы не рассматриваем здесь самый общий возможный случай графов, ограничиваясь тем, что необходимо для наиболее важных примеров. Более широкий класс примеров, требующий дополнительных определений, будет рассмотрен в готовящейся статье. Заметим лишь, что теория фильтраций позволяет рассматривать конечные аппроксимации инвариантных мер «из бесконечности», т.е. с точки зрения убывающей последовательности хвостовых сигма-алгебр пространства путей, а не возрастающей последовательности конечных сигма-алгебр.

В 22 приводятся все нужные определения и обзор относящихся к делу известных фактов. Множество инвариантных (центральных) мер - это проективный предел симплексов, а его вершины (граница Шоке) - это эргодические меры. Поэтому мы уделяем внимание геометрии проективных пределов, полезной и в других вопросах. Главная идея работы излагается в §3; сначала в элементарной геометрической форме определяется, что такое метрика Канторовича на симплексе, и затем дается определение так называемой внутренней метрики на проективном пределе симплексов. Это понятие позволяет разбить все эргодические меры на два класса - стандартные и нестандартные ${ }^{1)}$ Главный результат (основная теорема) приведен в §4: если выполнено условие равномерной компактности этажей (допредельных симплексов), то вся граница Шоке состоит из стандартных эргодических мер и может быть найдена как пополнение допредельных границ по внутренней метрике, иначе говоря, мы получаем параметризацию множества эргодических мер и интерпретацию параметров. Разумеется, общность этого подхода не дает надежд на точные формулы для распределений; такие формулы могут быть получены (и были получены) с учетом специфики (аналитической или вероятностной) в конкретных случаях. И хотя вряд ли можно рассчитывать на это в общем случае, тем не менее, выявление смысла параметров, естественной метрики на них и, главное, интерпретация их независимости открывают новый уровень в понимании задач об инвариантных мерах. В этой работе по соображениям экономии места мы лишь кратко упоминаем о примерах в 55 , имея в виду посвятить главным примерам другую работу.

В заключение прокомментируем два рисунка ${ }^{2)}:$ первый - граф диаграмм Юнга, т. е. граф ветвления неприводимых представлений бесконечной группы финитных подстановок. Этот граф стандартен в смысле этой работы - все эргодические меры на пространстве его путей стандартны, и они и составляют список неразложимых характеров этой группы. Но доказать это и получить полный список мер очень непросто. Впервые его аналитически нашел Тома [5]. Позже в работе [6] была реализована идея эргодического метода и был получен тот же список с объяснением комбинаторного и представленческого смысла параметров, чего не было в [5], а еще позже в работе [16] (см. также [8]) этот результат был получен методами, которые можно назвать операторными. Метод настоящей работы призван дать первое чисто комбинаторное доказательство

1) В предварительных работах [1], [2] мы использовали термины «гладкие» и «негладкие» меры, графы и т. д., следуя традициям теории $C^{*}$-алгебр, но предлагаемая здесь терминология кажется более уместной.

2) Рисунки выполнены П. Б. Затицким. 


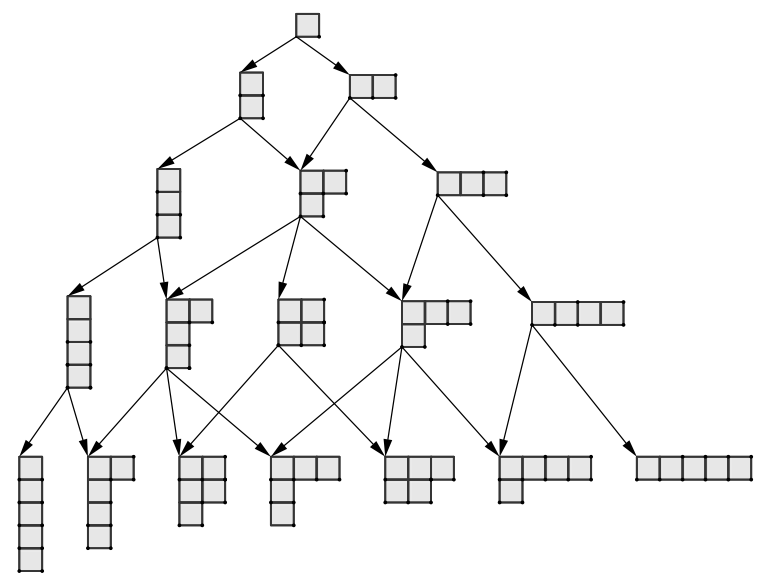

Рис. 1. Граф диаграмм Юнга

теоремы о мерах (о характерах), не использующее ни аналитической, ни алгебраической техники, а опирающееся только на комбинаторику графа Юнга. Главное состоит в том, что полнота списка (т. е. доказательство того, почему частоты определяют однозначно эргодическую меру) получается априори из теоремы о компактности. А граф на втором рисунке, граф неупорядоченных пар (вершины следующего этажа суть всевозможные неупорядоченные пары вершин данного этажа), - пример нестандартного графа. Он в простейшей форме иллюстрирует идею так называемой «башни мер», введенной в [4]. Разнообразные задачи об инвариантных мерах для ручных разбиений (т. е. монотонных пределов конечных разбиений) могут быть сведены к задачам на графах. Примеры нестандартных графов часто встречаются в теории динамических систем, в теории роста случайных конфигураций, в моделях статфизики.

\section{§2. Основные понятия}

2.1. Марковский компакт и пространство путей градуированного графа. Мы будем рассматривать задачу об инвариантных вероятностных мерах относительно некоторого отношения эквивалентности на пространстве траекторий топологического марковского компакта или на пространстве путей градуированного графа. Отношение эквивалентности будет задано с помощью аппроксимации этого отношения финитными отношениями эквивалентности.

Основное пространство, где будут заданы меры - это односторонний топологический марковский компакт $\mathscr{X}$. Такой компакт определяется набором пространств состояний, т. е. совокупностью конечных множеств $\left\{X_{n}\right\}, n=0,1, \ldots$, $X_{0}=\{\varnothing\}$, и набором 0-1-матриц $M_{n}=\left\{\epsilon_{i, j}\right\}, i \in X_{n-1}, j \in X_{n}, n=1, \ldots$, имеющих порядки $\left|X_{n-1}\right| \times\left|X_{n}\right|$ без нулевых строк и столбцов. Элементы марковского компакта $\mathscr{X}$ суть произвольные бесконечные последовательности (траектории) $\left\{x_{n}\right\}, n=0,1, \ldots$, элементов конечных множеств $X_{n}, x_{n} \in X_{n}$, удовлетворяющих условию допустимости, состоящему в точности в том, что для любой пары соседних элементов $x_{n-1}, x_{n}, n=1, \ldots$, последовательности должно выполняться условие $\epsilon_{x_{n-1}, x_{n}}=1$. Топология и сигма-алгебра $\mathfrak{A}$ борелевских множеств в пространстве $\mathscr{X}$ определяются естественным образом, поскольку 


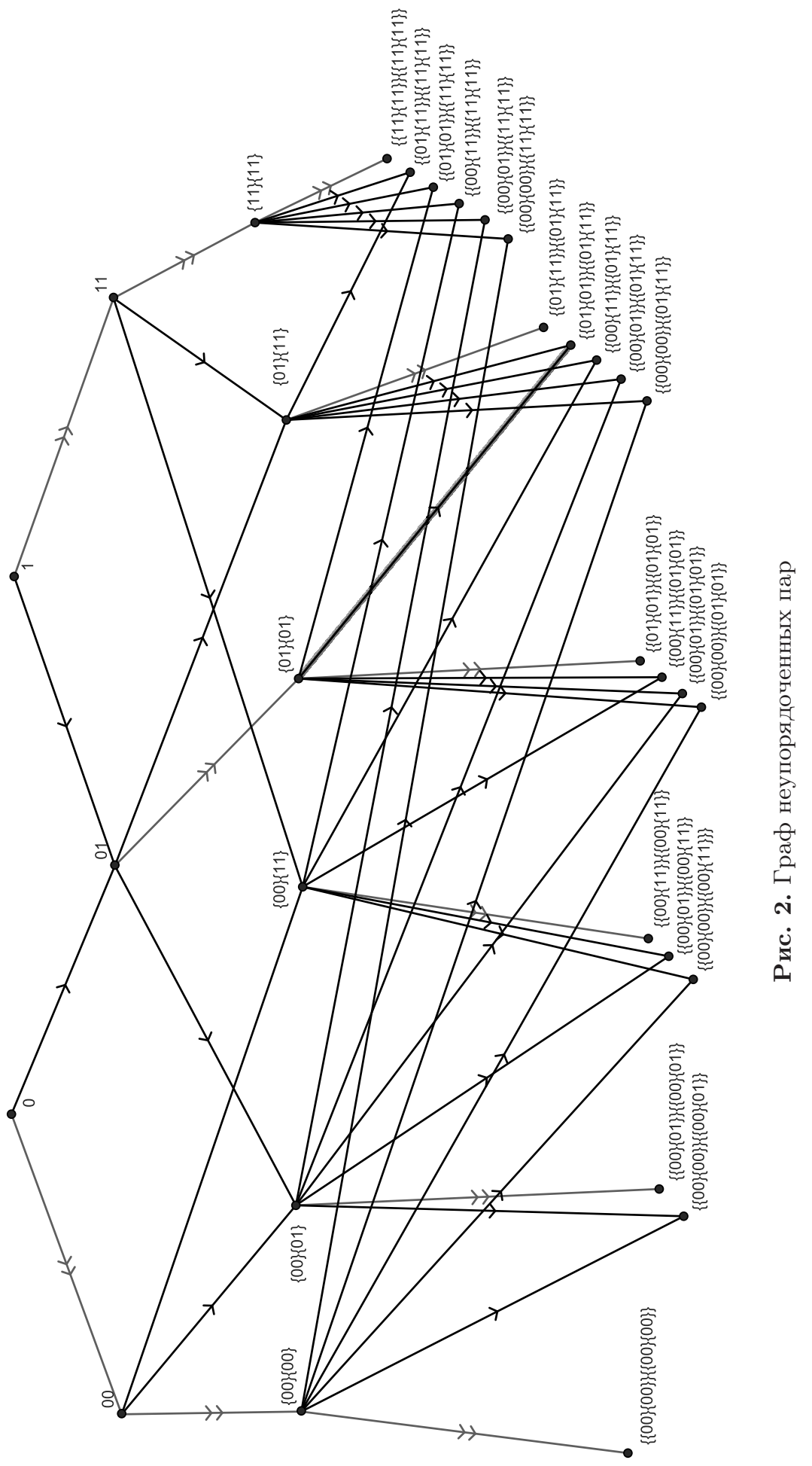


пространство $\mathscr{X}$ очевидным образом является обратным (проективным) пределом последовательности конечных пространств $\mathscr{X}_{n}$, состоящих из траекторий длины $n$, удовлетворяющих тому же условию допустимости, с проекциями забывания последней координаты $\mathscr{X}_{n} \rightarrow \mathscr{X}_{n-1}$.

Очевидно, что совокупность приведенных данных $\left\{X_{n} ; M_{n}\right\}_{n \geqslant 0}$ определяет в то же время и другой объект: бесконечный локально конечный $\mathbb{N}$-градуированный граф Г - так называемую диаграмму Браттели. При этом марковский компакт, определенный выше, отождествляется с пространством бесконечных путей этого графа. Пространство всех бесконечных путей в графе обозначается через $T(\Gamma) \sim \mathscr{X}$. Диаграммы Браттели и их комбинаторика, в свою очередь, теснейшим образом связаны с теорией локально полупростых алгебр и $A F$-алгебр, но здесь мы не останавливаемся на этом.

Таким образом, имеется возможность трактовать все последующее не только в терминах марковских компактов, естественных для вероятностных формулировок, но и в терминах градуированных графов $\Gamma$, их этажей $\Gamma_{n} \sim X_{n}$, $n=0,1, \ldots$, и их вершин $x_{n} \sim \gamma_{n}$, конечных или бесконечных путей $\left\{t_{n}=x_{n}\right\}$ и т. д. Поскольку эти два языка почти тавтологически совпадают (достаточно повернуть на 90 градусов изображение графа, и мы получим схему марковской цепи), мы используем далее оба языка одновременно, не опасаясь разночтений ${ }^{1)}$. Язык графов используется в основном для комбинаторного анализа приложений, которые и были основным источником обсуждаемых задач для автора. При этом выяснилось, что появляющиеся примеры, идущие из теории диаграмм Браттели, мало исследованы в рамках теории марковских компактов, динамических систем и теории процессов и, наоборот, полезные конструкции из теории динамических систем лишь сейчас находят адекватные применения в алгебраической теории.

Замечание 1. Удобно принять следующее соглашение: никакие две различные вершины одного и того же этажа графа (соответственно два или несколько элементов некоторого пространства состояний марковского компакта) не могут иметь одинаковые множества предшествующих вершин (т.е. в матрицах $M_{n}$ нет одинаковых строк). Это условие в дальнейшем обеспечит невырожденность внутренней метрики. Необременительность этого условия состоит в том, что если имеются такие вершины, то их можно заменить одной, но при этом изменить кратность ребер, умножая их на число объединенных вершин; проделав такую замену со всеми вершинами, мы получим новый граф, для которого условие выполнено.

Наиболее важные для дальнейшего понятия - хвостовое отношение эквивалентности на марковском компакте или на пространстве путей графа и хвостовые фильтрации на этих пространствах. Два пути или две последовательности в марковском компакте называются $n$-конфинальнымми, если они совпадают, начиная с уровня $n$; классы эквивалентных $n$-конфинальных последовательностей конечны. Измеримое (т. е. задаваемое как разбиение на прообразы борелевски измеримой функции) разбиение пространства путей на эти классы обозначим через $\xi_{n}$. Два пути конфинальны, если они $n$-конфинальны при некотором $n$. Классы конфинальности задают отношение эквивалентности, которое

1) В нынешней терминологии имеется тенденция, идущая от теории диаграмм Юнга, называть вершины графа $\Gamma$, т. е. состояния марковской цепи, диаграммами, а пути - таблицами, но мы здесь избегаем этого. 
будем называть хвостовым и обозначать через $\xi(\mathscr{X})$ или $\xi(\Gamma)$. Разбиение на классы конфинальности $\bigcap_{n} \xi_{n}$, вообще говоря, не является измеримым разбиением, но оно гиперконечно, т. е. по определению является пределом борелевских отношений с конечными классами эквивалентности. Сигма-алгебру множеств, измеримых относительно разбиения $\xi_{n}$ (т. е. множеств, содержащих вместе с данным путем все эквивалентные ему относительно разбиения $\xi_{n}$ ), обозначим через $\mathfrak{A}_{n}$. Сигма-алгебры $\mathfrak{A}_{n}$ убывают по $n$, начальная сигма-алгебра совпадает со всей сигма-алгеброй: $\mathfrak{A}_{0}=\mathfrak{A}$. Убывающие последовательности сигма-алгебр в пространствах с мерой или в топологических пространствах называют фильтрачиями; определенную выше последовательность $\left\{\mathfrak{A}_{n}\right\}$ мы назовем хвостовой фильтрацией на пространстве путей графа или в марковском компакте. Эти понятия и их свойства, изучаемые с 70-х гг. (см. [3], [4]), будут существенными в данной работе.

2.2. Дополнительные структуры на пространствах путей. На описанных объектах можно определить важные дополнительные структуры; мы будем их рассматривать позже и только кратко опишем их сейчас.

1. На конечном множестве ребер, ведущих в данную вершину, может быть определен линейный порядок; если он избран для всех вершин, то это позволяет определить лексикографический порядок на классах конфинальных путей и затем ввести частичное преобразование путей: переход от данного пути к следующему в смысле лексикографического порядка, так называемый адический сдвиг. Мы не будем использовать здесь это понятие, полезно лишь то, что хвостовое отношение эквивалентности есть не что иное, как траекторное разбиение для адического сдвига.

2. Другая дополнительная структура - коцикл копереходных вероятностей: с каждой вершиной $\gamma_{n} \in \Gamma$ (или координатой $x_{n} \in X_{n}$ ) связывается вероятностный вектор $\left\{\lambda_{\gamma_{n}}^{\gamma_{n-1}}\right\}$, где $\gamma_{n-1}$ пробегает все вершины из $\Gamma_{n-1}$, которые соединены с вершиной $\gamma_{n}$. Этот вектор трактуется как вектор копереходных вероятностей марковской цепи, т. е. как $\operatorname{Prob}\left(x_{n-1} \mid x_{n}\right)$.

В этой статье мы будем рассматривать эту структуру только в главном частном случае, канонически связанном с графом и марковским компактом; а именHо:

копереходные вероятности $\left\{\lambda_{\gamma_{n}}^{\gamma_{n-1}}\right\}$ (для пары $\left.\left(x_{n-1}, x_{n}\right)\right)$ пропорииональны числу всех путей, ведущих в $x_{n-1}$ из начальной вершины.

Меры с такими копереходными вероятностями называются центральнъми (инвариантными) мерами, см. ниже. Однако метод этой статьи без изменений применим к случаю общих переходных вероятностей (т. е. к теории квазиинвариантных мер). Число путей, ведущих из $\varnothing$ в вершину $\gamma$, обычно обозначается через $\operatorname{dim} \gamma$ (поскольку это размерность некоторого модуля).

3. Матрицы $M_{n}$, определяющие марковский компакт, могут иметь целые неотрицательные координаты, что соответствует мультиграфу (графу с кратными ребрами); в этом случае пространство путей есть последовательность ребер, а не вершин. Все основные теоремы верны и в этом случае. Можно рассматривать более общий контекст, когда кратности могут быть даже вещественными неотрицательными числами, которые соответствует весам ребер и путей.

Заметим, что все указанные дополнительные структуры могут быть формально описаны с помощью матриц $M_{n}$ более общего вида и истолкованы в 
терминах локально полупростых алгебр и $A F$-алгебр, ассоциированных с диаграммами Браттели; мы не останавливаемся здесь на этом.

2.3. Постановка задачи об инвариантных мерах. Перейдем к изложению основной задачи.

Пусть задан марковский компакт $\mathscr{X}$ (или пространство путей диаграммы Браттели $T(\Gamma))$; множество всех вероятностных борелевских мер $\operatorname{Meas}(\mathscr{X})$ на нем есть аффинный компактный (в слабой топологии) симплекс, экстремальные точки которого суть $\delta$-меры. Поскольку $\mathscr{X}$ есть обратный (проективный) предел конечных пространств (а именно пространств конечных путей), то, очевидно, и $\operatorname{Meas}(\mathscr{X})$ является обратным пределом конечномерных симплексов $\widehat{\Sigma}_{n}$, каждый из которых является множеством формальных выпуклых комбинаций конечных путей (или просто множеством вероятностных мер на этих путях), ведущих из начальной вершины в вершины этажа $n, n=1,2, \ldots$, а проекции $\hat{\pi}_{n}: \widehat{\Sigma}_{n} \rightarrow \widehat{\Sigma}_{n-1}$ соответствуют «забыванию» последней вершины пути. Каждая мера определяется своими конечномерными проекциями на цилиндрические множества (т. е. является так называемой цилиндрической мерой). Нас будут интересовать лишь центральные (инвариантные) меры, которые образуют подмножество в $\operatorname{Meas}(\mathscr{X})$.

Определение 1. Вероятностная борелевская мера $\mu$ на марковском компакте (= на пространстве путей графа) называется иентралъной, если для любой вершины произвольного этажа проекция этой меры на подалгебру цилиндрических множеств конечных путей, заканчивающихся в данной вершине, есть равномерная мера на этом (конечном) множестве путей.

Другие, эквивалентные, определения центральной меры $\mu \in \operatorname{Meas}(\mathscr{X})$ :

1. Условная мера относительно меры $\mu$ при условии фиксации «хвоста» бесконечного пути, проходящего через данную вершину, т. е. условная мера на элементах разбиения $\xi_{n}$ (см. разд. 2.1), есть равномерная мера на начальных отрезках пути для любой вершины.

2. Мера инвариантна относительно любого адического сдвига (т. е. при любом выборе порядков на ребрах). Иногда это выражают следующими словами: мера инвариантна относительного хвостового отношения эквивалентности.

Термин «центральная мера» связан с тем, что в приложении к теории следов алгебр и характеров групп меры с этими свойствами определяют следы на алгебрах (соответственно характеры на группах). В теории стационарных (однородных) топологических марковских цепей центральные меры называются мерами с наибольшей энтропией.

Множество центральных мер на марковском компакте $\mathscr{X}$ (на пространстве $T(\Gamma)$ путей графа Г) будем обозначать через $\operatorname{Inv}(\mathscr{X})$ или $\operatorname{Inv}(\Gamma)$. Очевидно, что центральные меры образуют выпуклое слабо замкнутое подмножество симплекса всех мер на марковском компакте: $\operatorname{Inv}(\mathscr{X}) \subset \operatorname{Meas}(\mathscr{X})$. Множество центральных мер $\operatorname{Inv}(\mathscr{X})$ также есть симплекс, который естественно представляется в виде проективного предела последовательности конечномерных симплексов выпуклых комбинаций равномерных мер на классах $n$-конфинальности. Более подробно:

Предложение 1. Симплекс иентральных мер представим в следующем виде:

$$
\operatorname{Inv}(\mathscr{X})=\lim _{\longleftarrow}\left(\Sigma_{n} ; p_{n, m}\right)
$$


นภU

$$
\Sigma_{1} \leftarrow \Sigma_{2} \leftarrow \cdots \leftarrow \Sigma_{n} \leftarrow \Sigma_{n+1} \leftarrow \cdots \Sigma_{\infty} \equiv \operatorname{Inv}(\mathscr{X}),
$$

где $\Sigma_{n}$ есть симплекс формальных выпукльх комбинаций вершин $n$-го этажса $\Gamma_{n}$ (т.е. точек из $\left.X_{n}\right)$, а проекиия $p_{n, n-1}: \Sigma_{n} \rightarrow \Sigma_{n-1}$ сопоставляет вершине $\gamma_{n} \in \Gamma_{n}$ выпуклую комбинащию $\sum \lambda_{\gamma_{n-1}}^{\gamma_{n}} \delta_{\gamma_{n-1}} \in \Sigma_{n-1}$, где числа $\lambda_{\gamma_{n-1}}^{\gamma_{n}}$ определяются однозначно из условия их пропорииональности числу путей, ведущих из $\varnothing$ в $\gamma_{n-1}$ (которое обозначено, как ужее упоминалось, через $\left.\operatorname{dim} \gamma_{n-1}\right)^{1)}$. Общий вид проеки,ии: $p_{n, m}=\prod_{i=m}^{n+1} p_{i, i-1}, m>n$.

Доказательство. Множество всех борелевских вероятностных мер на пространстве путей есть симплекс, являющийся проективным пределом симплексов, порожденных пространствами конечных путей длины $n$; это следует из того, что само пространство путей есть проективный предел с очевидными проекциями (забывание последнего ребра). Пространство инвариантных мер есть, таким образом, слабозамкнутое выпуклое подмножество этого симплекса, и мы покажем, что оно есть также проективный предел симплексов (то, что множество инвариантных мер относительно счетного отношения эквивалентности всегда есть так называемый симплекс Шоке, хорошо известно, см. далее и, например, [9]). Проекция $\mu_{n}$ любой инвариантной меры $\mu$ на конечный цилиндр этажа $n$ есть инвариантная мера относительно замен начальных отрезков путей и потому лежит в определенном выше симплексе, а указанные проекции сохраняют инвариантность; поэтому $\left\{\mu_{n}\right\}$ есть точка проективного предела. Остается лишь заметить, что мера однозначно определяется своими проекциями, в силу чего между точками проективного предела и множеством инвариантных мер $\operatorname{Inv}(\Gamma)$ установлена биекция.

Напомним, что барицентрические координаты точки симплекса $\Sigma_{n}$ можно рассматривать как вероятностную меру на множестве его вершин. Замечание 1 означает, что разные вершины графа соответствуют разным вершинам симплекса.

Экстремальные точки симплекса $\operatorname{Inv}(\Gamma)$ инвариантных мер на всем пространстве путей $T(\Gamma)$ есть неразложимые инвариантные меры, т. е. меры, не являющиеся нетривиальной выпуклой комбинацией других инвариантных мер. Отсюда и из теоремы о разложении на эргодические компоненты мер, инвариантных относительно гиперконечного отношения эквивалентности, следует, что неразложимая мера эргодична (т. е. не существует инвариантных множеств промежуточной меры). Именно эти меры представляют для нас главный интерес, так как остальные меры суть их выпуклые комбинации, возможно, континуальные. Множество центральных эргодических мер марковского компакта $\mathscr{X}$ (графа Г) обозначим через $\operatorname{Erg}(\mathscr{X})$ или $\operatorname{Erg}(\Gamma)$.

Проблема 1. Oписать все чентральные эргодические меры для данного марковского компакта (соответственно все неразложимые централъные меры для данного графа). Более определенным является вопрос, для каких марковских компактов (или графов) множсество иентральных эргодических мер имеет аналитическое описание в терминах комбинаторных характеристик

1) В общем (нецентральном) случае коэффициенты $\lambda$ суть копереходные вероятности (см. выше).

2 Функциональный анализ и его приложения, т. 48, вып. 4 
этого компакта (графа) и каковы эти характеристики, а также в каких случаях такой ответ невозможен. Этими характеристиками могут быть свойства последовательности матрии $\left\{M_{n}\right\}$, задающих компакт (граф), частотьи, спектры и т.д. В первом случае, а именно когда такое описание возможно, компакт (граф) далее называется стандартным.

Простейший пример стандартного графа - граф Паскаля, а множество эргодических мер дается теоремой де Финетти, параметры - частоты (одномерные распределения).

Эта проблема включает задачу описания унитарных факторпредставлений конечного типа дискретных локально конечных групп, задачу описания конечных следов некоторых $C^{*}$-алгебр, задачу о границе-вход и границе-выход по Дынкину (см. [12]); она имеет теснейшие связи с задачами о границах Мартина, Пуассона-Фюрстенберга и др. Ответ на вопрос, поставленный в проблеме, может быть как «ручным» (существует борелевская параметризация эргодических мер или факторпредставлений конечного типа), так и «диким» (такой параметризации не существует). В теории представлений, как хорошо известно с 50-х гг., такая ситуация имеет место в теории неприводимых представлений групп и алгебр. Однако это случается, правда, значительно реже, и в теории факторпредставлений. Но во многих классических ситуациях ответ «ручной», что априори совсем не очевидно.

Например, характеры бесконечной симметрической группы, т. е. инвариантные меры на путях графа Юнга (см. рис. 1), имеют хорошую параметризацию, и это глубокий результат, а для графа неупорядоченных пар (см. рис. 2) хорошей параметризации нет. Подчеркнем, что представление симплекса $\operatorname{Inv}(\Gamma)$ в виде проективного предела симплексов существенно зависит от аппроксимации, т. е. от структуры марковского компакта (графа). Очевидно, что и решение поставленного вопроса зависит от аппроксимации. Дело в том, что аппроксимацию можно менять, не меняя запаса инвариантных мер, который определяется лишь хвостовым отношением эквивалентности. Зависимость наших ответов от аппроксимации будет обсуждаться позже (см. замечание в последнем параграфе о теореме о лакунарном изоморфизме). Но поскольку в реальных задачах аппроксимация явно присутствует уже в самой постановке, то и ответ нужно формулировать в ее терминах. См. примеры далее.

2.4. Геометрические формулировки. Мы напомним некоторые известные геометрические формулировки, поскольку далее будем использовать удобный язык выпуклой геометрии.

1. Множество всех борелевских вероятностных мер на сепарабельном компакте, инвариантных относительно действия счетной группы (или отношения эквивалентности) образует симплекс, т. е. сепарабельный аффинный компакт в слабой топологии с единственным разложением всякой точки компакта в интеграл по мере на множестве экстремальных точек ${ }^{1)}$. Множество эргодических мер есть граница Шоке, т. е. множество экстремальных точек симплекса; оно всегда имеет тип $G_{\delta}$-множества.

1) Теорема Шоке о разложении точек выпуклого компакта в интеграл по вероятностной мере на множестве экстремальных точке есть не очень сложное усиление предшествующей принципиальной теоремы Крейна-Мильмана о том, что выпуклый аффинный компакт есть слабое замыкание множества выпуклых комбинаций экстремальных точек. 
2. Используется следующая терминология: симплекс Шоке называется симплексом Полсена [10], если его граница Шоке слабо плотна в нем, и симплексом Бауэра (Bauer), если граница замкнута. Между этими двумя случаями возможны промежуточные.

3. Проективный предел симплексов (см. ниже) есть симплекс Полсена тогда и только тогда, когда при любом $n$ объединение проекций множеств вершин симплексов с бо́льшими номерами на $n$-й симплекс всюду плотно. Универсальность симплекса Полсена была замечена и доказана много позже ([11], [13]):

Предложение 2. Все сепарабельные симплексы Полсена топологически изоморфны как афбинные компакты; этот единственный с точностъю до изоморфизма симплекс универсален в смысле теории моделей ${ }^{1)}$.

Нетрудно проверить, что всякий проективный предел симплексов возникает при изучении квазицентралъных мер на пространстве путей графа или марковских мер, имеющих заданные копереходные вероятности (см. выше). Но мы далее рассматриваем лишь центральные меры, т. е. весьма специальную систему проекций в определении проективного предела. Однако по существу особых различий в методе изучения общего случая по сравнению со случаем центральных мер нет. Мы вернемся к этому в другом месте.

Добавим еще два простых факта, следующих из определений.

4. Любая центральная эргодическая мера на марковском компакте (пространстве путей графа) является марковской относительно структуры марковского компакта (эргодичность существенна).

5. Относительно любой центральной эргодической меры хвостовая фильтрация является полуоднородной, что и означает равномерность почти всех условных мер для любого разбиения $\xi_{n}, n=1, \ldots$

Метрической теории полуоднородных фильтраций будет посвящена отдельная работа.

2.5. Экстремальность точек проективного предела и эргодичность марковских мер. Мы приведем критерий эргодичности меры в терминах общих проективных пределов симплексов, иначе говоря, дадим критерий экстремальности точки проективного предела симплексов.

Пусть задан произвольный проективный предел $\Sigma_{\infty}$ симплексов

$$
\Sigma_{1} \leftarrow \Sigma_{2} \leftarrow \cdots \leftarrow \Sigma_{n} \leftarrow \Sigma_{n+1} \leftarrow \cdots
$$

с аффинными проекциями $p_{n, n-1}: \Sigma_{n} \rightarrow \Sigma_{n-1}, n=1, \ldots$ (общий вид проекции $p_{m, n}: \Sigma_{m} \rightarrow \Sigma_{n}$ приведен выше).

Рассмотрим элемент проективного предела $x_{\infty} \in \Sigma_{\infty}$; он определяет и определяется последовательностью $\left\{x_{n}\right\}$ своих проекций, $x_{n} \in \Sigma_{n}, n=1, \ldots$, на конечномерные симплексы. Зафиксируем натуральные $n<m$, разложим (единственным образом) элемент $x_{m}$ как точку симплекса $\Sigma_{m}$ в выпуклую комбинацию его экстремальных вершин $e_{i}^{m}$ :

$$
x_{m}=\sum_{i} c_{m}^{i} \cdot e_{i}^{m}, \quad \sum_{i} c_{m}^{i}=1, c_{m}^{i} \geqslant 0,
$$

1) То есть любой сепарабельный симплекс может быть инъективно отображен в симплекс Полсена и изоморфизм любых двух изоморфных граней симплекса продолжается до автоморфизма всего симплекса. 
и обозначим через $\mu_{m}=\left\{c_{m}^{i}\right\}_{i}$ меру на вершинах симплекса, отвечающую этому разложению. Спроектируем эту меру $\mu_{m}$ на симплекс $\Sigma_{n}, n<m$, и обозначим проекцию через $\mu_{m}^{n}$ - это мера на симплексе $u$, тем самым, случайная точка cuмnлекса; заметим, что эта мера, вообще говоря, не сосредоточена на вершинах симплекса $\Sigma_{n}$.

Предложение 3 (экстремальность точки проективного предела симплексов). Экстремальность точки $x_{\infty}=\left\{x_{n}\right\}$ предельного симплекса $\Sigma_{\infty}$ равносильна слабой сходимости последовательности мер $\mu_{n}^{m}$ nри $m \rightarrow \infty \kappa$ дельтамере $\delta_{x_{n}}$ при всех значениях $n$ :

$$
\forall \epsilon>0 \quad \forall n \quad \exists K=K_{\epsilon, n} \quad \forall m>K \quad \mu_{n}^{m}\left(V_{\epsilon}\left(\mu_{n}\right)\right)>1-\epsilon,
$$

где $V_{\epsilon}(\cdot)$ есть эпсилон-окрестность точки в обычной топологии (например, евклидовой).

Достаточно использовать непрерывность в топологии проективного предела разложения произвольной точки $x_{\infty}$ по экстремальным и спроектировать это разложение на конечномерные симплексы; тогда для экстремальных точек, и только для них, последовательность проекций должна сходится к дельта-мере.

Нетрудно пересказать этот критерий для нашего случая $\Sigma_{\infty}=\operatorname{Inv}(\Gamma)=$ $\operatorname{Inv}(\mathscr{X})$. Теперь удобно рассматривать координаты (проекции) центральной меры $\mu_{\infty}$ не как точки конечномерных симплексов, а как меры на их вершинах $\left\{\mu_{n}\right\}$ (что, конечно, одно и то же). Тогда меры $\mu_{m}^{n}$ надо рассматривать как меры на вероятностных векторах, занумерованных вершинами симплекса, а меру $\mu$ на марковском компакте $\mathscr{X}$ (или на $T(\Gamma))$ как точку предельного симплекса $\operatorname{Inv}(\Gamma)$. Сформулированный критерий заключается в том, что $\mu$ является эргодической мерой (т. е. экстремальной точкой в $\operatorname{Inv}(\Gamma))$ тогда и только тогда, когда последовательность мер $\mu_{m}^{n}$ стремится слабо при $m \rightarrow \infty$ к мере $\mu_{n}$ (как мере на вершинах симплекса $\left.\Sigma_{n}\right)$ при всех $n$.

В вероятностных терминах наше утверждение есть топологическая версия теоремы о сходимости мартингалов по мере и выглядит совсем просто: для любого $n$ условное распределение координаты $x_{n}$ при фиксации координаты $x_{m}$, $m>n$, стремится по вероятности к безусловному распределению координаты $x_{n}$, когда $m \rightarrow \infty$.

Согласно этому предложению, чтобы найти конечномерные проекции эргодических мер, нужно перебрать все дельта-меры, которые являются слабыми пределами мер $\mu_{n}^{m}$ при $m \rightarrow \infty$. Но, конечно, такой способ неэффективен и тавтологичен. Более эффективный эргодический метод для своего обоснования требует усиления утверждения, а именно, сходимости не по мере, а почти всюду, т. е. индивидуальной эргодической теоремы или поточечной сходимости мартингалов (см. [14], [6]). Содержание этой статьи заключается в эффективизации эргодического метода с помощью нового типа сходимости мартингалов.

\section{§3. Внутренняя метрика и корневая норма на проективных пределах симплексов}

3.1. Метрики на конечных множествах и корневая норма. Мы начнем со следующего простого замечания, играющего, тем не менее, фундаментальную роль. А именно, дадим элементарное определение метрики Канторовича 
в самом простом конечномерном случае с чисто геометрических позиций. Рассмотрим конечное множество $E=\left\{e_{i}\right\}$ и построим конечномерный симплекс $\Sigma(E)$ формальных выпуклых комбинаций точек этого множества. Предположим, что на множестве $E$, т. е. на множестве вершин $\left\{e_{i}\right\}$ симплекса, задана некоторая метрика $\rho$. Эту метрику можно, например, продолжить на каждое из ребер как евклидову с длиной ребра, равной расстоянию между его концами. Можно ли естественно продолжить эту метрику на грани старших размерностей и на весь симплекс и каков запас этих продолжений?

Теорема 1 (определение продолжения метрики). Существует функториальное продолжение $\bar{\rho}$ метрики $\rho$, заданной на множестве вершин симплекса, на весь симплекс. Имеется в виду, что операция продолжения, определяемая ниже, есть бунктор из категории конечных метрических пространств в категорию аффинных симплексов вещественного векторного пространства с метрикой.

Это продолэсение есть не что иное, как метрика Канторовича (или транспортная метрика) на симплексе; эта метрика определяет норму в афбинной оболочке симплекса (норму Канторовича-Рубинштейна), которая является максимальной в классе всех возможных продолжений метрики с множества вершин на афбинную оболочку.

Доказательство. Продолжение метрики и норма определяются следующим образом. Каждой точке симплекса $x \in \Sigma$ можно сопоставить совокупность ее барицентрических координат, определяющую единственную вероятностную меру $\nu_{x}$ на множестве вершин $e_{i}, i=1, \ldots, n$, этого симплекса, барицентром которой эта точка является:

$$
x=\sum_{i} c_{i} e_{i}, \quad \nu_{x}=\left\{c_{i} \delta_{e_{i}}\right\}, \quad c_{i} \geqslant 0, \sum_{i} c_{i}=1 .
$$

Рассмотрим расстояние $k_{\rho}$ по Канторовичу между двумя мерами $\nu_{x}$ и $\nu_{y}$, отвечающими точкам $x$ и $y$ симплекса, оно и объявляется продолжением $\bar{\rho}$ метрики $\rho$ на весь симплекс. Приведем определение метрики Канторовича в наших обозначениях:

$$
\bar{\rho}(x, y) \equiv k_{\rho}\left(\nu_{x}, \nu_{y}\right)=\min _{\psi}\left\{\sum_{i, j} \psi_{i, j} \rho\left(e_{i}, e_{j}\right): \psi=\left\{\psi_{i, j}\right\}, \sum_{j} \psi_{i j}=c_{i}, \sum_{i} \psi_{i j}=d_{j}\right\} .
$$

Легко видеть, что на множестве вершин симплекса метрика $\bar{\rho}$ совпадает с исходной: $\bar{\rho}\left(e_{i}, e_{j}\right)=\rho\left(e_{i}, e_{j}\right)$. На ребрах симплекса это обычная евклидова метрика с заданным расстоянием между концами ребер, а на гранях большей размерности она существенно зависит от метрики на вершинах. Удобно погрузить симплекс в его аффинную оболочку, а затем выбирать в качестве начала координат линейного пространства сумму вершин симплекса. Тогда метрика на симплексе определяет норму (Канторовича-Рубинштейна) на аффинной оболочке, которая выражается формулой

$$
\left\|c-c^{\prime}\right\|=k_{r}\left(c, c^{\prime}\right), \quad\|\lambda c\|=|\lambda|\|c\|,
$$

где $c, c^{\prime}$ - две точки конуса, натянутого на симплекс, а корректность определения следует из того, что норма не зависит от представления элемента в виде разности точек конуса. 
Максимальность этой нормы среди всех норм на аффинной оболочке, для которых $\left\|\delta_{x}-\delta_{y}\right\|=\rho(x, y)$, следует из того, что единичный шар в этой норме является выпуклой оболочкой элементов вида $\delta_{x}-\delta_{y}$ (простых корней, см. далее $)^{1)}$.

Таким образом, имеется конечно-параметрическое семейство норм в $\mathbb{R}^{n}$, каждая из которых определяется своими значениями на разностях координатных векторов. Это семейство и есть семейство метрик Канторовича и норм Канторовича-Рубинштейна в простейшем (конечном) случае транспортной задачи. Норма Канторовича-Рубинштейна и ее сопряженная (липшицева норма) изучались во многих работах для бесконечномерного, континуального, случая, однако геометрия шаров в этих нормах (в конечномерном пространстве $\mathbb{R}^{n}$ ) не менее интересна, чем геометрия шаров и норм в $l^{p}$ или других традиционных норм. Как ни странно, эти нормы, насколько известно автору, не упоминаются в учебниках по оптимизации или по выпуклой геометрии. См. об этом работу [17], посвященную совсем другой теме.

Единичный шар в этих метриках есть весьма любопытный выпуклый центрально-симметричный многогранник. Для размерности $d=2$ это произвольный центрально-симметричный шестиугольник, т. е. норма гексагональна; для $d=3$ это многогранник, комбинаторно эквивалентный кубу. Общий случай более сложен и, видимо, не изучен. Метрику $\bar{\rho}$ на симплексе можно назвать также корневой метрикой, отвечающей метрике $\rho$ на вершинах симплекса. Если $\rho=\delta_{x, y}=1, x \neq y$, то это корневая метрика в смысле алгебры Ли $A_{n}{ }^{2)}$.

Замечание 2. Из определения непосредственно вытекает важное свойство монотонности метрик:

$$
\bar{\rho}_{n-1}\left(p_{n, n-1} x, p_{n, n-1} y\right) \leqslant \bar{\rho}_{n}(x, y), \quad x, y \in \Sigma_{n},
$$

3.2. Определение внутренней метрики. Мы переходим к индуктивному построению основного понятия - внутренней метрики на проективных пределах симплексов и, в частности, на симплексе инвариантных мер марковской цепи. Для этого мы итерируем метрику Канторовича по возрастающей размерности. Предположим, что задан проективный предел симплексов $\lim _{n}\left\{\Sigma_{n}, p_{n, m}\right\}$.

Определение 2. Зафиксируем произвольную метрику $\rho_{k}$ на множестве вершин симплекса $\Sigma_{k}$ при некотором $k \geqslant 1$ и ее продолжение на весь симплекс $\Sigma_{k}$, которое будем обозначать теперь той же буквой $\rho_{k}$. Определим по индукции метрики на всех последующих симплексах $\Sigma_{n}, n \geqslant k$, следующим образом: вершины симплекса $\Sigma_{n+1}, n \geqslant k$, в силу определения проективного предела проектируются в некоторые точки предыдущего симплекса $\Sigma_{n}$, на котором метрика уже определена. Примем ее за метрику на вершинах симплекса $\Sigma_{n+1}$ и продолжим указанным способом до метрики на всем симплексе, обозначая также через $\rho_{n+1}$.

1) Максимальность - не единственное свойство метрики Канторовича, выделяющее ее из множества всех возможных продолжений метрики с множества вершин симплекса на весь симплекс; другое ее свойство используется в предложении 5 .

2) Связь этой нормы с теорией простых алгебр Ли состоит в следующем. Пусть наш симплекс лежит в алгебре Картана алгебры Ли $A_{n}$ и порожден простыми корнями $\epsilon_{i, i+1}$, $i=1, \ldots, n-1$, и $\epsilon_{i, 1}$, на которых задана дельта-метрика. Тогда единичным шаром в этой норме служит выпуклая оболочка всех положительных и отрицательных корней; эту норму естественно назвать корневой нормой, она инвариантна относительно группы Вейля. Рассматривалась ли она подробно (см. [15])? 
В дальнейшем мы будем считать фиксированной начальную метрику $\rho_{k}$ и обозначать итерированные внутренние метрики через $\left\{\rho_{n}\right\}_{n=1}^{\infty}$. Ниже мы покажем, что основное свойство (стандартность) от выбора метрики $\rho_{k}$ не зависит ${ }^{1}$.

Мы получили семейство метрик на симплексах и, в частности, на совокупности вершин каждого из них. Покажем, как распространить метрики на несвязное объединение вершин всех симплексов. Сначала определим расстояние между вершиной $x$ симплекса $\Sigma_{n}$ и вершиной $f$ симплекса $\Sigma_{n+1}$ как расстояние по внутренней метрике на $\Sigma_{n}$ между проекцией вершины $f$ на $\Sigma_{n}$ и вершиной $x$. Далее, расстояние между вершинами $e \in \Sigma_{n}$ и $f \in \Sigma_{m}, m>n$, определим как минимум сумм расстояний $\rho\left(e_{i}, e_{i+1}\right)$ по парам вершин соседних этажей по всем цепочкам вершин $e=e_{n}, e_{n+1}, \ldots, e_{m-1}, e_{m}=f$ промежуточных этажей с конечной точкой $f$ и начальной $e$. Можно было бы определить метрику на всем объединении симплексов, но нам это не понадобится.

Определение внутренней метрики на вершинах симплекса позволяет перенести ее на вершины графа и на состояния марковской цепи. При этом, согласно замечанию 1, каждой вершине симплекса соответствует только одна вершина графа, и поэтому образ метрики на вершинах графа есть метрика (а не полуметрика).

Замечание 3. Общее определение внутренних метрик (или полуметрик) естественно давать не для множеств вершин симплексов или вершин графа на каждом из этажей, а для пространства последовательностей вершин (ребер) графа, т. е. на пространстве путей графа или траекторий марковской цепи. Это делается с помощью универсального приема «переноса (полу)метрики», подобного описанному выше. Результатом итераций будет некоторая полуметрика на пространстве бесконечных путей, которая факторизуется до метрики на пространстве центральных мер, а точнее, на пространстве дизъюнктных борелевских (но, вообще говоря, незамкнутых) носителей центральных эргодических мер. Поскольку во многих приложениях достаточно рассматривать внутренние метрики как уже определенные и компактифицировать объединение пространств вершин, мы откладываем общее определение до другого раза.

3.3. Независимость от начальной метрики. Независимость понятия стандартности экстремальной точки от начальной метрики, которая определяет внутреннюю метрику, вытекает из следующего предложения.

Предложение 4. Пусть на произвольном конечном множестве $S$ заданы две метрики $\rho, \rho^{\prime}$, удовлетворяющие соотношению

$$
\rho(x, y) \leqslant r \cdot \rho^{\prime}(x, y)
$$

при всех $x, y \in S$, где $r$ - положительная константа. Тогда для соответствующих метрик Канторовича на симплексе мер $\Sigma(S)$ выполнено такое же неравенство:

$$
k_{\rho}\left(\mu_{1}, \mu_{2}\right) \leqslant r \cdot k_{\rho^{\prime}}\left(\mu_{1}, \mu_{2}\right) \quad \text { для любъх } \mu_{1}, \mu_{2} \in \Sigma(S) .
$$

1) Несколько отклоняясь от нашей основной линии, заметим, что определенная выше внутренняя метрика имеет следующую интерпретацию: это есть метрика на пространстве градуированных конечномерных модулей, каковыми являются вершины данного этажа диаграммы Браттели. Эта интерпретация важна для алгебраических приложений. Интересно сравнить ее с другими метриками на пространствах модулей над алгебрами, если такие метрики имеются. 
Доказательство. Воспользуемся теоремой двойственности, выражающей значение метрики Канторовича как супремум по единичному шару интегралов от функций Липшица по разности мер:

$$
k_{\rho}\left(\mu_{1}, \mu_{2}\right)=\sup _{u \in \operatorname{Lip}_{\rho, 1}} \int_{S} u(x) d\left(\mu_{1}-\mu_{2}\right) .
$$

Очевидно, что из неравенства для метрик вытекает следующее соотношение между единичными шарами пространств функций Липшица:

$$
\operatorname{Lip}_{\rho, 1} \subset r \cdot \operatorname{Lip}_{\rho^{\prime}, 1},
$$

откуда сразу следует нужное неравенство.

Следствие 1. Асимптотические (по размерности) свойства внутренних метрик, определяемых по начальным метрикам, такие, как сходимость последовательностей и др., не зависят от начальной метрики.

Действительно, в силу доказанного предложения неравенства между метриками в точности сохраняются при итерациях.

В общем случае начальная метрика определяется на пространстве путей (конечных или нет), начинающихся в нулевой вершине, а последовательные итерации переносят ее на пути, начинающиеся с 1-го, 2-го и т. д. этажей (см. замечание 3). Вывод и доказательство независимости предельного поведения этих метрик от начальной остается верным и в этом случае.

\section{§4. Стандартность и равномерная компактность}

\section{1. Стандартные экстремальные точки и стандартные эргодиче-} ские меры. Тривиальной экстремальной точкой проективного предела симплексов является последовательность $\left\{x_{n}\right\}$, состоящая из экстремальных точек допредельных симплексов, $x_{n} \in \operatorname{Ex}\left(\Sigma_{n}\right)$; такие точки не представляют интереса, если они и существуют. С другой стороны некоторые экстремальные точки проективных пределов удовлетворяют гораздо более сильным условиям концентрации, чем в условии экстремальности (предложение 4). Для нас важен случай, когда последовательность проекций $\left\{x_{n}\right\}$ такова, что расстояния по некоторой метрике до множества вершин симплекса стремятся к нулю. Для того чтобы точно сформулировать, что значит такая сходимость, необходимо ввести метрику на симплексах всех размерностей по некоторому общему правилу ${ }^{1)}$. Именно такое определение было дано выше - это внутренняя метрика.

Это позволяет разделить множество экстремальных точек и, в частности, множество центральных эргодических мер на марковском компакте или на пространствах путей графа на два класса.

Ввиду важности определения стандартности мы сформулируем его отдельно для произвольного проективного предела симплексов, а затем специально конкретизируем для мер на марковских компактах и для мер на путях графа. Не составляет труда понять, что эти формулировки выражают в разных терминах одно и то же свойство ${ }^{2}$.

1) При этом универсальность такой сходимости возможна, если определение функториально.

${ }^{2)}$ В этой работе мы не останавливаемся на том, как связаны все эти формулировки с общим определением стандартности фильтраций, как и на том, почему стандартность в смысле 
Определение 3. 1. Рассмотрим проективный предел $\lim \left\{\Sigma_{n}, p_{m, n}\right\} \equiv \Sigma_{\infty}$ симплексов $\Sigma_{n}$. Экстремальная точка $x_{\infty} \in \Sigma_{\infty}$ с проекциями $\left\{x_{n} \in \Sigma_{n}\right\}$ называется стандартной, если $\lim _{n \rightarrow \infty} \rho_{n}\left(x_{n}, \operatorname{Ex}\left(\Sigma_{n}\right)\right)=0$, т. е. расстояние от нее до границы симплекса по внутренней метрике стремится к нулю. Заметим, что экстремальность точки уже следует из этого условия. Это свойство не зависит от выбора начальной метрики.

2. Центральная мера $\mu$ на пространстве путей графа $\Gamma$ называется стандартной, если существует такая последовательность вершин $\gamma_{n} \in \Gamma_{n}$, что $\lim \mu_{n}\left(V_{\epsilon}\left(\gamma_{n}\right)\right)=1$ для любого $\epsilon>0$, где $V_{\epsilon}(\gamma)$ есть $\epsilon$-окрестность в метрике $\rho_{n}$. Это свойство можно назвать свойством асимптотической кониентрации меры на путях или законом больших чисел. Оно не зависит от выбора начальной метрики.

3. Мера с максимальной энтропией (центральная мера) на марковском компакте $\mathscr{X}$ называется стандартной, если она обладает следующим свойством: расстояние по метрике $\rho_{n}$ между условными распределениями на $X_{n}$ (при фиксации условий на $\left.X_{n+1}\right)$ стремится по мере к нулю. Это свойство усиливает теорему о сходимости мартингалов, поскольку сходимость мер по внутренней метрике означает равномерность обычной сходимости конечномерных распределений по размерности; оно также не зависит от выбора начальной метрики. Экстремальные точки проективного предела симплексов (=центральные эргодические меры), не являющиеся стандартными, называются нестандартными. Проективный предел симплексов (соответственно граф, марковский компакт) называется стандартным, если все его экстремальные точки (эргодические меры) стандартны.

В теории фильтраций ([3], [4]) понятие стандартности было введено как неочевидное обобщение свойства независимости; например, однородная эргодическая фильтрация является фильтрацией сигма-алгебр прошлых последовательности независимых случайных величин (схемы Бернулли) тогда и только тогда, когда она стандартна (критерий стандартности в [3]). В общем неоднородном случае и даже в полуоднородном имеется много неизоморфных стандартных фильтраций, которые устроены достаточно сложно. Но общее свойство, роднящее их с независимостью, состоит в том, что для всех таких фильтраций есть «дополнительный» базис, т. е. хорошо структурированное относительно фильтрации семейство измеримых множеств, порождающих полную сигма-алгебру. В теории стационарных процессов класс стандартных фильтраций прошлого есть подкласс класса регулярных фильтраций ${ }^{1)}$. Его естественно сравнить со свойством Орнштейна очень слабой бернуллиевости (very weak Bernoulli) [16], с которым оно находится в общем положении (т. е. ни одно не вытекает из другого), но они сходны по характеру определений: различие состоит в метриках, используемых на пространствах условных мер.

Само условие стандартности можно также назвать условием кониентрации по внутренней метрике, поскольку речь идет о том, что проекции меры концентрируются около некоторой точки границы в этой метрике или что пути графа

этих определений есть свойство фильтрации, не зависящее от способа ее реализации в виде хвостовой фильтрации. Это будет исследовано в уже упоминавшейся готовящейся статье о метрической и комбинаторной теории фильтраций.

1) Регулярность (или колмогоровость) фильтрации означает тривиальность пересечения последовательности $\Sigma$-алгебр. 
при больших $n$ лежат в малой $\rho$-окрестности одной вершины. Это свойство иногда называется свойством «сущестования предельной формы» (limit shape), но точный смысл этих слов зависит от метрики. Наконец, это свойство не без оснований может быть названо обобщенным законом больших чисел. Это особенно ясно видно из формулировки 2. Однако следует помнить о роли метрики в теоремах типа «limit shape».

Мы выделили класс проективных пределов (графов, марковских компактов), для которых все центральные эргодические меры стандартны, и приходим к следующей принципиальной задаче.

Проблема 2. Описать стандартные проективные пределы симплексов (стандартные графы, стандартные марковские компакты), например, в терминах матрии, $\left\{M_{n}\right\}$ или в иных терминах. Асимптотический характер этой задачи (состоящий в том, что ответ не зависит от изменения конечного числа начальных матрич) позволяет надеяться лишь на достаточные условия стандартности.

Мы увидим позже, что стандартными оказываются многие классические примеры (графы Паскаля, Юнга, случайные блуждания на группах и др.). В то же время существуют графы, не являющиеся стандартными, представляющие собой типичный случай (см. рис. 2). Предлагаемая процедура позволяет не только проверять стандартность, когда она есть, но получать параметризацию эргодических мер.

Определение 4. Последовательность $\left\{x_{n}\right\}$ вершин симплексов $\Sigma_{n}$ (или последовательность $\left\{x_{n}\right\}$ вершин графа $\left.\Gamma_{n}\right)$ называется регулярной, если существует стандартная экстремальная точка $x_{\infty}$ проективного предела (соответственно стандартная центральная мера на путях графа), последовательность проекций которой на симплексы $\Sigma_{n}$ сближается по внутренней метрике с последовательностью $x_{n}$.

Регулярные последовательности разбиваются на классы, параметризуемые всеми стандартными экстремальными точками. Легко убедиться, что верен следующий критерий регулярности:

Предложение 5. Последовательность $\left\{x_{n}\right\}$ регулярна, если она сходится в себе по внутренней метрике (см. определение в конще разд. 3.2).

4.2. Равномерная компактность. Начнем с простого утверждения (доказанного еще в [4])

Лемма 1. Если число вершин на всех уровнях графа Г (соответственно число точек в пространствах состояний $X_{n}$ ) ограничено константой, не зависящей от $n$, то граф (марковский компакт) стандартен, т.е. все иентральнъе эргодические меры стандартны.

Доказательство. Заметим, что среднее расстояние между точками относительно итераций метрики $\rho_{n}$ стремится к нулю, поскольку число точек не растет, а хотя бы для одной пары точек на следующем шаге расстояние уменьшается в фиксированное число раз и при этом ни одно из расстояний не может оставаться постоянным в силу эргодичности, а стремление к нулю равномерно по всем мерам на данном компакте.

Интересно, какова минимальная скорость роста числа вершин, при которой возникнет нестандартность. 
Будем рассматривать внутреннюю метрику на множествах $\Gamma_{n}$, т. е. вершинах этажей графа, или на множествах $X_{n}$ состояний марковской цепи.

Определение 5. Будем говорить, что для семейства $\left\{\Gamma_{n}\right\}$ имеет место равномерная компактность по внутренней метрике, если для любого $\epsilon>0$ число точек в $\epsilon$-сети для всех множеств $\Gamma_{n}$ равномерно ограничено по $n$.

Если это условие выполнено для множеств $\Gamma_{n}$, т. е. для множеств вершин симплексов, то оно выполняется и для совокупности самих симплексов, снабженных внутренней метрикой.

Следствие 2. Если в $\operatorname{Inv}(\Gamma)$ существует нестандартная экстремальная точка, то семейство $\left\{\Gamma_{n}\right\}$ не является равномерно компактным.

Действительно, компактность означала бы, что при всех $\epsilon>0$ имеется равномерно ограниченное множество точек в $\epsilon$-сети на всех этажах, а по лемме 1 отсюда следовала бы стандартность любой экстремальной точки.

На множество стандартных экстремальных точек (стандартных эргодических мер) можно продолжить по непрерывности внутреннюю метрику.

Теорема 2. Если семейство симплексов $\left\{\Sigma_{n}\right\}$ (эквивалентно, семейство множеств их вершин) равномерно компактно относительно $n$ по внутренней метрике, то проективный предел симплексов (соответственно диаграмма Браттели, марковский компакт) стандартен. В этом случае проективный предел есть симплекс Бауэра, т.е. его граница Шоке замкнута, и внутренняя топология на гранище Шоке совпадает со слабой топологией. Совокупность экстремальных точек совпадает с множеством пределов регулярных последовательностей вершин.

Доказательство. В силу равномерной компактности по внутренней метрике из следствия 2 вытекает, что всякая экстремальная точка стандартна. Но всякая стандартная экстремальная точка есть предел регулярной последовательности вершин. Пополнение по внутренней метрике пространства классов эквивалентных последовательностей, таким образом, совпадает с множеством всех экстремальных точек. Поэтому граница Шоке компактна. Отсюда же следует совпадение топологий, поскольку тождественное отображение симплекса с внутренней топологией в него же со слабой топологией, очевидно, непрерывHO.

Коротко говоря, равномерная компактность совокупности симплексов по внутренней метрике означает компактность множества экстремальных точек и проективного предела симплексов по внутренней метрике.

Отметим, что множество стандартных экстремальных точек может быть пустым и при этом симплекс может быть или не быть бауэровским (т. е. со слабо компактной границей Шоке). Например, существуют примеры, в которых граница Шоке замкнута и даже одноточечна, но экстремальная точка не является стандартной и нужной компактности симплексов во внутренней топологии нет.

Вывод. Предлагается способ решения проблемы о перечислении центральных мер, состоящий в выяснении, имеет ли место равномерная компактность семейства вершин симплексов, или вершин графа, или состояний марковского компакта по внутренней метрике. Если равномерная компактность этих семейств имеет место, то пополнение объединения этих семейств по этой метрике есть множество всех инвариантных эргодических мер. В этом случае все эти 
меры стандартны и удовлетворяют условию концентрации. Непосредственная параметризация множества эргодических мер вытекает из построения. Данный вывод сохраняет силу и для задачи перечисления мер с заданными копереходами (т. е. квазиинвариантных мер), т. е. для описания границ-вход и границвыход, границ Мартина и др.

\section{§5. Комментарии и примеры}

5.1. Зависимость от аппроксимации и лакунарный изоморфизм. Напомним, что по теореме о лакунарном изоморфизме (в однородном случае см. [4]) любая фильтрация лакунарно стандартна, т. е. содержит стандартную подпоследовательность. Отсюда следует, что если допустить переход от данного графа (данного марковского компакта) к графу (компакту), в котором пропускаются некоторые этажи (возможно, бесконечное их число), а пропущенные вершины и ребра компенсируются кратностью новых ребер, то нестандартная центральная мера может стать стандартной. Эта операцию назовем операиией разрежения. Эта операция меняет внутреннюю топологию: в разреженном графе топология ослабляется. Тем самым, вообе говоря, расширяется запас стандартных экстремальных точек. Поэтому операция разрежения может превратить нестандартный граф в стандартный, если разрежение делает одновременно все меры стандартными ${ }^{1)}$. Однако практически операция разрежения весьма неэффективна, и получение стандартного графа из нестандартного такой ценой неоправданно. Например, для фильтраций, имеющих положительную энтропию, необходимое разрежение растет суперэкспоненциально. Кроме того, по сути вопроса естественно связывать параметризацию центральных мер с исходным, а не разреженным графом.

5.2. Примеры вычислений. Ясно, что практический вывод из сделанных рассмотрений состоит в том, что необходимо вычислить внутреннюю метрику на этажах и проверить, есть ли равномерная компактность. Это чисто комбинаторная задача, иногда простая, а иногда не очень простая. Пополнение по внутренней метрике в случае компактности дает явную параметризацию экстремальных точек.

Теорема 3. Обобщенный граф Паскаля $\mathbb{Z}_{+}^{d+1}$ размерности $d$ является стандартным. Список инвариантных эргодических мер есть список бернуллиевских мер, параметризующихся точками единичного $(d-1)$-мерного симплекса $($ при $d=2$ это теорема де Финетти). Граф Юнга (см. рис. 1) такюсе стандартен.

Обобщенный граф Паскаля размерности $d-$ это решетка $\mathbb{Z}_{+}^{d+1}$ с градуировкой по сумме координат; для обычного графа Паскаля $d=1$. Подсчет внутренней метрики в этом случае кратко намечен в [1]. В этом случае достаточно манипуляций с биномиальными или мультиномиальными коэффициентами. Доказательство стандартности дает еще одно доказательство обобщенной теоремы де Финетти. Подробные вычисления мы оставляем до подробной статьи о примерах.

Стандартность графа Юнга косвенно следует из теоремы Тома (т. е. из списка характеров), но есть все основания надеяться на возможность прямой проверки

1) Неясно, существует ли граф, для которого такое разрежение невозможно. Если таковой существует, то, скорее всего, это случайный градуированный граф. 
условия компактности по внутренней метрике и, тем самым, на первое чисто комбинаторное доказательство теоремы Тома. Помимо использования формул для размерности (формулы крюков, например), полезна аппроксимация самих графов: свойство равномерной компактности в данном случае можно получить из аппроксимации графа Юнга многомерными графами Паскаля растущей размерности. Параметр Тома пробегает симплекс двусторонних неотрицательных сходящихся рядов с суммой, не большей 1.

Скорее всего, стандартность имеет место и для гораздо более общего случая, охватывающего все предыдущие - для графов, являющихся диаграммами Хассе дистрибутивных решеток с конечным числом образующих. Например, многомерные графы Юнга входят в этот класс. Любопытно, что на роль параметров во всех этих случаях претендуют независимые частоты некоторых (минимальных бесконечных) идеалов и заявленная выше обобщенная независимость и есть определенная независимость этих частот. Из стандартности (т. е. из компактности множества стандартных экстремальных точек) следует компактность множества этих частот. Причина, по которой во всех этих случаях имеет место равномерная компактность (см. выше), лежит в следующем устройстве графов: все вершины, предшествующие любой данной вершине, близки по внутренней метрике и эта близость тем больше, чем больше уровень этажа, которому принадлежит вершина.

Именно отсутствие этого свойства характерно для имеющихся примеров нестандартных графов. Для графа неупорядоченных пар (см. рис. 2) нет ответа на простые вопросы, связанные с внутренней метрикой, например, на вопрос, каков рост эпсилон-энтропии. Все эти вопросы тесно связаны с теорией так называемой башни мер [4].

\section{ЛитерАТУРА}

[1] A. M. Vershik, Intrinsic metric on graded graphs, standardness, and invariant measures, Зап. научн. сем. ПОМИ, 421 (2014), 58-67.

[2] A. Vershik, Smooth and non-smooth AF-algebras and a problem on invariant measures, http://arxiv.org/abs/1304.2193.

[3] А. М. Вершик, Убывающие последовательности измеримых разбиений и их приложения, Докл. АН СССР, 193 (1970), 748-751.

[4] А. М. Вершик, Теория убъвающих последовательностей измеримых разбиений, Алгебра и анализ, 6:4 (1994), 1-68.

[5] E. Thoma, Die Unzerlegbaren positiv-definiten Klassenfunktionen der abzählbar unendlichen Simmetrischen Gruppe, Math. Z., 85:1 (1964), 40-61.

[6] А. М. Вершик, С. В. Керов, Асимптотическая теория характеров симметрической групnы, Функц. анализ и его прил., 15:4 (1981), 15-27.

[7] А. Ю. Окуньков, О представлениях бесконечной симметрической группь, Зап. научн. сем. ПОМИ, 240 (1997), 166-228.

[8] Г. И. Ольшанский, Унитарные представления $(G, K)$-пар, связанных с бесконечной симметрической группой $S(\infty)$, Алгебра и анализ, 1:4 (1989), 178-209.

[9] Р. Фелпс, Лекиии о теоремах Шоке, Мир, М., 1968.

[10] E. T. Poulsen, A simplex with dense extreme points, Ann. Inst. Fourier (Grenoble), 11 (1961), 83-87.

[11] J. Lindenstrauss, G. Olsen, Y. Sternfeld, The Poulsen simplex, Ann. Inst. Fourier (Grenoble), 28:1 (1978), 91-114. 
[12] Е. Б. Дынкин, Началъное и финальное поведение траекторий марковских проиессов, УМН, 26:4(160) (1971), 153-172.

[13] W. Lusky, The Gurarij spaces are unique, Arch. Math. (Basel), 27:1 (1976), 627-635.

[14] А. М. Вершик, Опиисание инвариантых мер для действий некотрых бесконечномерных групп, Докл. АН СССР, 218:4 (1974), 749-752.

[15] Э. Б. Винберг, Инвариантные нормы в компактных алгебрах Ли, Функц. анализ и его прил., 2:2 (1968), 89-90.

[16] D. Ornstein, Ergodic Theory, Randomness, and Dynamical Systems, Yale Mathematical Monographs, vol. 5, Yale Univ. Press, New Haven-London, 1974.

[17] J. Melleray, F. V. Petrov, A. M. Vershik, Linearly rigid metric spaces and the embedding problem, Fund. Math., 199:2 (2008), 177-194.

[18] А. М. Вершик, Инвариантные мерь, градуированные графь, фильтрачии. Курс лекций в Лаборатории им. П. Л. Чебышева, 2014.

Санкт-Петербургское отделение Математического института им. В. А. Стеклова РАН

Поступило в редакцию Санкт-Петербургский государственный университет

8 августа 2014 г. Институт проблем передачи информации e-mail: avershik@gmail.com 